On the Convergence of the Tapia Indicators in the Absence of Strict Complementarity

A.S. El-Bakry

R.A. Tapia

Y. Zhang

September, 1993

TR93-39 



\title{
On the Convergence of the Tapia Indicators in the Absence of Strict Complementarity
}

\author{
A. S. El-Bakry, R. A. Tapia ${ }^{2}$ and Y. Zhang ${ }^{3}$
}

\begin{abstract}
The convergence and the convergence rate of the Tapia indicators were established in ElBakry [1] and EL-Bakry, Tapia, and Zhang [2] in the context of primal-dual interior-point method under the assumption of strict complementarity. In the current work we use a recent result of Monteiro and Wright, [10] to extend this theory to the case when strict complementarity does not hold.
\end{abstract}

Keywords: Interior-point methods, the Tapia indicator, Strict complementarity. Abbreviated Title: The Tapia Indicators for Degenerate Complementarity Problems. AMS(MOS) subject classifications: $65 \mathrm{~K}, 49 \mathrm{M}, 90 \mathrm{C}$

\footnotetext{
${ }^{1}$ Department of Mathematics, Faculty of Science, Alexandria University, Alexandria, Egypt and the Center for Research on Parallel Computations, Rice University, Houston, Texas, 77521-1892(elbakry@rice.edu). This author was supported in part by NSF Coop. Agr. No. CCR-8809615 and AFOSR 89-0363 and the REDI Foundation

${ }^{2}$ Department of Computational and Applied Mathematics and the Center for Research on Parallel Computations, Rice University, Houston, Texas, 77521-1892(ratorice.edu). This author was supported in part by NSF Coop. Agr. No. CCR-8809615, AFOSR 89-0363, DOE DEFG05-86ER25017 and ARO 9DAAL03-90-G-0093

${ }^{3}$ Department of Mathematics and Statistics, University of Maryland Baltimore County, Baltimore, MD 21228 , and visiting member of the Center for Research on Parallel Computations, Rice University, Houston, Texas, 775211892(yzhang@math.umbc,edu). This author was supported in part by NSF DMS-9102761 and DOE DE-FG0293ER25171
} 


\section{Introduction}

In this paper we consider the monotone linear complementarity problems

$$
\text { Find }(x, y) \in \mathbf{R}^{n} \times \mathbf{R}^{n} \text { such that } y=M x+q, \quad(x, y) \geq 0, \quad x^{T} y=0,
$$

where $q \in \mathbf{R}^{n}$, and $M \in \mathbf{R}^{n \times n}$. Many optimization problems can be formulated as monotone linear complementarity problems including the linear programming problem. We write (1.1) as

$$
F(x, y) \equiv\left(\begin{array}{c}
M x-y+q \\
X Y e
\end{array}\right)=0
$$

and

$$
(x, y) \geq 0
$$

where $X=\operatorname{diag}(x)$ and $Y=\operatorname{diag}(y)$. Let $\mathcal{S}$ denotes the solution set of problem (1.1). A solution pair $(x, y)$ is said to satisfy strict complementarity if in addition to complementarity $X Y e=0$, it satisfies $x+y>0$. For problem (1.1), we will be interested in the following three subsets of the index set $\{1, \ldots, n\}$

$$
\begin{aligned}
\mathcal{B} & =\left\{i: x_{i}^{*}>0 \text { for at least one }\left(x^{*}, y^{*}\right) \in \mathcal{S}\right\} \\
\mathcal{N} & =\left\{i: y_{i}^{*}>0 \text { for at least one }\left(x^{*}, y^{*}\right) \in \mathcal{S}\right\} \\
\mathcal{J} & =\left\{i: x_{i}^{*}=y_{i}^{*}=0 \text { for all }\left(x^{*}, y^{*}\right) \in \mathcal{S}\right\}
\end{aligned}
$$

It is clear that $\mathcal{B}, \mathcal{N}$, and $\mathcal{J}$ form a partition of $\{1, \ldots, n\}$.

In general, for monotone linear complementarity problems solutions that satisfy strict complementarity may not exist. However, for linear programming problems, the existence of solutions that satisfy strict complementarity was proved by Goldman and Tucker [6]. In this case, all solutions in the relative interior of the solution set satisfy strict complementarity, see for example El-Bakry, Tapia and Zhang [2] and we have $\mathcal{J}=\emptyset, \mathcal{B} \cup \mathcal{N}=\{1, \ldots, n\}$, and $\mathcal{B} \cap \mathcal{N}=\emptyset$.

For linear programming many indicators function for identifying members of $\mathcal{B}$ and $\mathcal{N}$ have been proposed. This information can be used to computational advantages in interior-point methods. A thorough study for this issue can be found in El-Bakry, Tapia, and Zahng [2]. In this paper we are concerned with identifying members of $\mathcal{B}, \mathcal{N}$, and $\mathcal{J}$. We consider three indicators for that purpose. The variables used as indicators, the primal-dual indicator, and the Tapia indicators. The variables used as indicators are perliaps the most widely used indicators in computational optimization. The primal-dual indicators are also used for the purpose of identifying the above subgroups of variables, particularly in the context of primal-dula interior-point methods, see for example Gay [4], Ye [12], and Lustig [8]. Monteiro and Wright [10] proposed the use of the primal-dual indicator in the context of degenerate monotone complementarity problems. The Tapia indicators were proposed by Tapia [11] for the identification of active and inactive constraints 
for general constrained optimization problems. The Tapia indicators were used by El-Bakry [1] and Mehrotra [9]. Their convergence properties, in primal-dual interior-point methods for linear programming, was studied in El-Bakry, Tapia, and Zhang [2]. The convergence of the Tapia indicators in the absence of strict complementarity has not been studied.

The motivation for studying the Tapia indicators in this context is that solutions that satisfy strict complementarity may not exist in general complementarity or optimization problems. One example is the monotone linear complementarity problem (1.1). Another interesting example is a class of problems arising from the control of some elasto-dynamic systems, see for example [5].

In this paper, we prove that the Tapia indicators corresponding to variables with indices in $\mathcal{J}$, i.e. variables that do not satisfy strict complementarity at every solution $\left(x^{*}, y^{*}\right) \in \mathcal{S}$, actually converge. This result is a direct consequence of the work of Monteiro and Wright [10]. It should be noted that this result holds only for feasible-iterate algorithms, whereas the convergence of the Tapia indicators when strict complementarity holds does not require feasibility. We also establish a convergence rate of the Tapia indicators in this context. Finally we study the convergence and convergence rate for the variables used as indicators and the primal-dual indicators.

This paper is organized as follows; in Section 2, a generic algorithm is introduced and the Tapia indicators and their convergence properties for linear programming problems are stated. We also define two widely used indicators in interior-point methods, namely the variables used as indicators and the primal-dual indicator. In Section 3 we establish a convergence result for the Tapia indicators corresponding to variables with indices in $\mathcal{J}$. We also study the convergence and the convergence rate for the three indicators. A result concerning the performance of interior-point methods in the existence of solutions that do not satisfy strict complementarity is also presented. We present some numerical experiments in Section 4. Final conclusions are given in Section 5.

\section{The Algorithm and Indicators}

A generic primal-dual Newton interior-point method for problem 1.1 has the following structure.

\section{Algorithm 2.1 (Primal-Dual Interior-Point Method)}

Given a strictly feasible starting point $\left(x^{0}, y^{0}\right)$. For $k=0,1, \ldots$, do

1. Choose $\sigma^{k} \in(0,1)$ and set $\mu(x, y)=\sigma^{k} \frac{\left(x^{k}\right)^{T} y^{k}}{n}$.

2. Solve the following system for $\left(\Delta x^{k}, \Delta y^{k}\right)$ :

$$
F^{\prime}\left(x^{k}, y^{k}\right)(\Delta x, \Delta y)=-F\left(x^{k}, y^{k}\right)+\mu\left(x^{k}, y^{k}\right) \hat{e}
$$

3. Choose $\tau^{k} \in(0,1)$ and set the steplength $\alpha^{k}=\min \left(1, \tau^{k} \hat{\alpha}^{k}\right)$ where

$$
\hat{\alpha}^{k}=\frac{-1}{\min \left(\left(X^{k}\right)^{-1} \Delta x^{k},\left(Y^{k}\right)^{-1} \Delta y^{k},-\frac{1}{2}\right)} .
$$


4. Form the new iterate

$$
\left(x^{k+1}, y^{k+1}\right)=\left(x^{k}, y^{k}\right)+\alpha^{k}\left(\Delta x^{k}, \Delta y^{k}\right)
$$

Observe that since $\left(x^{0}, y^{0}\right)$ is strictly feasible, all the subsequent iterates are also strictly feasible. We make the following assumptions.

(A1) $\left(x^{k}\right)^{T} y^{k} \rightarrow 0$.

(A2) $x_{i}^{k} y_{i}^{k} \geq \bar{\gamma}\left(x^{k}\right)^{T} y^{k}$ for all $i=1, \ldots, n$ and for some positive constant $\bar{\gamma}$.

Now, we state three indicators, the variables used as indicators, the primal-dual indicator and, the Tapia indicators for problem (1.1).

\section{The Variables:}

$$
V\left(x^{k}\right)=x^{k} \text {, and } V\left(y^{k}\right)=y^{k} \text {. }
$$

The Primal-dual indicator:

$$
P D\left(x^{k}, y^{k}\right)=\left(Y^{k}\right)^{-1} x^{k}
$$

where $Y^{k}=\operatorname{diag}\left(y^{k}\right)$.

The Tapia indicators:

$$
T\left(x_{i}^{k}\right)=\frac{x_{i}^{k+1}}{x_{i}^{k}}
$$

and

$$
T\left(y_{i}^{k}\right)=\frac{y_{i}^{k+1}}{y_{i}^{k}},
$$

where $x_{i}^{k+1}=x_{i}^{k}+\alpha^{k} \Delta x_{i}^{k}$ and $y_{i}^{k+1}=y_{i}^{k}+\alpha^{k} \Delta y_{i}^{k}$.

For the sake of completeness we include El-Bakry, Tapia, and Zhang [2] convergence result for the Tapia indicators for linear programming problems.

Proposition 2.1 (El-Bakry-Tapia-Zhang) Let the sequence of iterates $\left\{\left(x^{k}, y^{k}\right)\right\}$ be generated by Algorithm 2.1 applied to the linear programming problem. Under the conditions

1. $\mathcal{S}$ is bounded,

2. $\left(x^{k}\right)^{T} y^{k} \rightarrow 0$,

3. $\frac{\min \left(X^{k} Y^{k} e\right)}{\left(x^{k}\right)^{T} y^{k}} \geq \gamma$ for all $k$ and some positive constant $\gamma$,

4. $\sigma^{k} \rightarrow 0$ and $\tau^{k} \rightarrow 1$ 
then for $i=1, \ldots, n$

$$
\lim _{k \rightarrow \infty} T\left(x_{i}^{k}\right)= \begin{cases}0 & \text { if } i \in \mathcal{N} \\ 1 & \text { if } i \in \mathcal{B}\end{cases}
$$

and

$$
\lim _{k \rightarrow \infty} T\left(y_{i}^{k}\right)= \begin{cases}0 & \text { if } i \in \mathcal{B} \\ 1 & \text { if } i \in \mathcal{N}\end{cases}
$$

where $x^{k+1}=x^{k}+\beta^{k} \Delta x$ and $y^{k+1}=y^{k}+\beta^{k} \Delta y$ for any $\beta^{k} \in\left[\alpha^{k}, 1\right]$, where $\alpha^{k}$ is given by Algorithm 2.1.

An example that demonstrates the behavior of the primal Tapia indicator for AFIRO, one of the Netlib [3] set of test problems, is given in Figure 1.

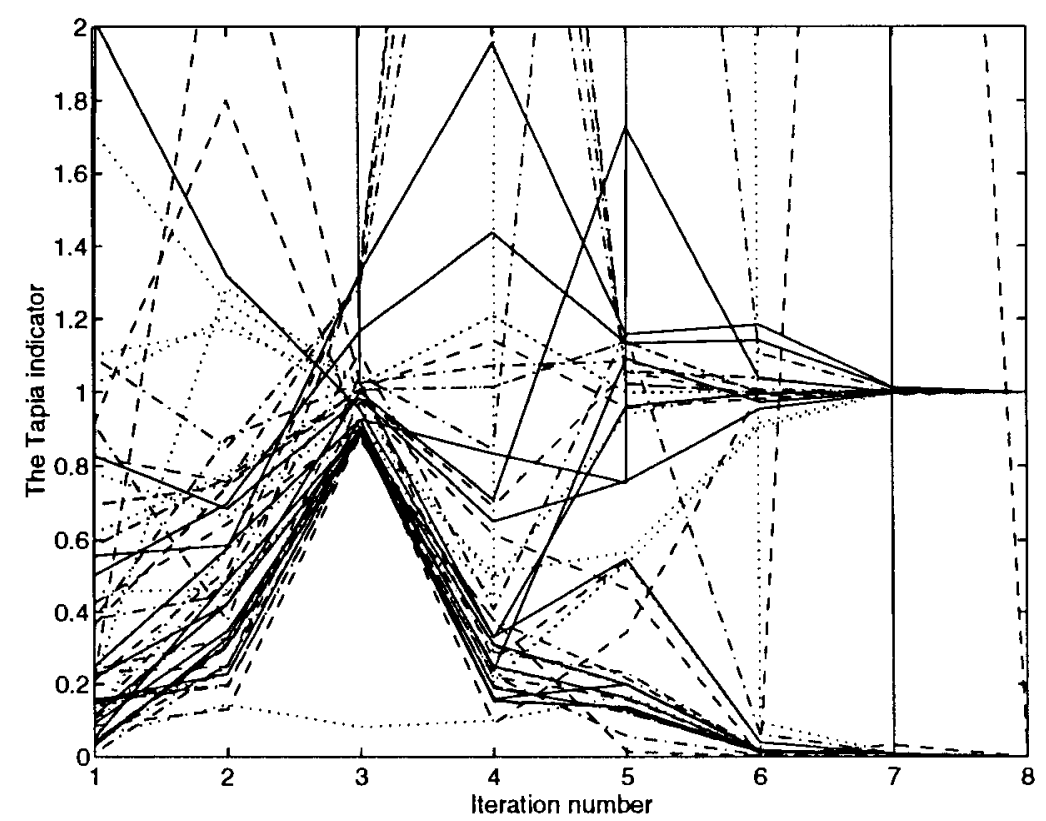

Figure 1: The Tapia primal indicator for all variables in AFIRO.

\section{Convergence in the Absence of Strict Complementarity}

One interesting property of the Tapia indicators is that their 0-1 separation property holds componentwise, i.e. if a pair $\left(x_{j}, y_{j}\right), j \in \mathcal{N}$ satisfies strict complementarity, then the 0 - 1 separation property holds for that pair even if other pairs do not satisfy strict complementarity.

In a recent paper, Monteiro and Wright [10] considered interior-point methods for degenerate monotone complementarity problems (problems of the form (1.1) where the solution set $\mathcal{S}$ may not 
contain a solution that satisfies strict complementarity). It is satisfying that as a direct consequence of their result, we are able to prove convergence of the Tapia indicators for pairs $\left(x_{j}, y_{j}\right), j \in \mathcal{J}$.

Lemma 3.1 Let $\left\{\left(x^{k}, y^{k}\right)\right\}$ be a sequence generated by Algorithm 2.1 and that Assumptions (A1)A(2) hold. Assume further that

1. the centering parameter is chosen such that $\sigma^{k} \rightarrow 0$.

2. $\lim \alpha^{k} \rightarrow \bar{\alpha} \in(0,1]$.

Then

$$
\lim _{k \rightarrow \infty} T\left(x_{i}^{k}\right)= \begin{cases}1-\bar{\alpha} & \text { if } i \in \mathcal{N} \\ 1-\frac{\bar{\alpha}}{2} & \text { if } i \in \mathcal{J} \\ 1 & \text { if } i \in \mathcal{B}\end{cases}
$$

Moreover

$$
\left|T\left(x_{i}^{k}\right)-1\right| \leq C\left(\sqrt{\left(x^{k}\right)^{T} y^{k}}+\sigma^{k}\right) \text { for } i \in \mathcal{B},
$$

and

$$
\left|T\left(x_{i}^{k}\right)\right| \leq C\left(\sqrt{\left(x^{k}\right)^{T} y^{k}}+\sigma^{k}\right) \text { for } i \in \mathcal{N}
$$

Proof: Let $i \in \mathcal{B}$, then

$$
\lim _{k \rightarrow \infty} \frac{x_{i}^{k+1}}{x_{i}^{k}}=\lim _{k \rightarrow \infty}\left(1+\alpha^{k} \frac{\Delta x_{i}^{k}}{x_{i}^{k}}\right) .
$$

But from Lemma 2.2 and Lemma 4.4. of Monteiro and Wright [10],

$$
x_{i}^{k} \geq C_{1} \text { and } \Delta x_{i}^{k}=O\left(\left[\left(x^{k}\right)^{T} y^{k}\right]^{1 / 2}+\sigma^{k}\right) \text { for } i \in \mathcal{B}
$$

where $C_{1}$ is some positive constant. Thus

$$
\lim _{k \rightarrow \infty} \frac{x_{i}^{k+1}}{x_{i}^{k}}=1,
$$

since $\left(x^{k}\right)^{T} y^{k} \rightarrow 0$ and $\sigma^{k} \rightarrow 0$ by the assumptions of the lemma. From the linearized complementarity

$$
\Delta x_{i} y_{i}+\Delta y_{i} x_{i}=-x_{i} y_{i}+\sigma x^{T} y
$$

we have

$$
\frac{x_{i}^{k+1}}{x_{i}^{k}}+\frac{y_{i}^{k+1}}{y_{i}^{k}}=\left(2-\alpha^{k}\right)+\alpha^{k} \sigma^{k} \frac{\left(x^{k}\right)^{T} y^{k}}{x_{i}^{k} y_{i}^{k}}
$$

Therefore

$$
\lim _{k \rightarrow \infty} \frac{y_{i}^{k+1}}{y_{i}^{k}}=1-\bar{\alpha} .
$$


From symmetry we have, for $i \in \mathcal{N}$,

$$
\lim _{k \rightarrow \infty} \frac{x_{i}^{k+1}}{x_{i}^{k}}=1-\bar{\alpha} .
$$

From Lemma 4.5 in Monteiro and Wright [10] we have

$$
\lim _{k \rightarrow \infty} \frac{\Delta x_{i}^{k}}{x_{i}^{k}}=-\frac{1}{2} \text { for } i \in \mathcal{J}
$$

Hence, for $i \in \mathcal{J}$, we have

$$
\begin{aligned}
\lim _{k \rightarrow \infty} \frac{x_{i}^{k+1}}{x_{i}^{k}} & =\lim _{k \rightarrow \infty}\left(1+\alpha^{k} \frac{\Delta x_{i}^{k}}{x_{i}^{k}}\right) \\
& =1-\frac{\bar{\alpha}}{2} .
\end{aligned}
$$

Now from Lemma 4.4. of Monteiro and Wright [10] we have, for $i \in \mathcal{B}$,

$$
\left|T_{i}\left(x^{k}\right)-1\right| \leq \alpha^{k}\left|\frac{\Delta x_{i}^{k}}{x_{i}^{k}}\right| \leq \bar{C}\left(\sqrt{\left(x^{k}\right)^{T} z^{k}}+\sigma^{k}\right) .
$$

It is easy to see that the same result is also true for $T_{i}\left(y^{k}\right)$ with $i \in \mathcal{N}$. Now we have, for $i \in \mathcal{N}$,

$$
\left|T_{i}\left(x^{k}\right)\right| \leq\left|T_{i}\left(y^{k}\right)-1\right|+O\left(\sigma^{k}\right)
$$

which implies that

$$
\left|T_{i}\left(x^{k}\right)\right| \leq C\left(\sqrt{\left(x^{k}\right)^{T} z^{k}}+\sigma^{k}\right) \text { for } i \in \mathcal{N}
$$

and completes the proof.

Lemma 3.1 states that the Tapia indicators converge if $\alpha^{k} \rightarrow \vec{\alpha}$. It is not clear, however, that the sequence $\left\{\alpha^{k}\right\}$ converges in many interior-point algorithms for problem (1.1). Nevertheless, $\left\{\alpha^{k}\right\}$ is usually constructed to be bounded in $(0,1)$. So $\lim \inf _{k \rightarrow \infty} \alpha^{k}$ exists. In this case a similar proof to that of Lemma 3.1 gives

$$
\lim \inf _{k \rightarrow \infty} T\left(x_{i}^{k}\right)= \begin{cases}1-\alpha^{\prime} & \text { if } i \in \mathcal{N} \\ 1-\frac{\alpha^{\prime}}{2} & \text { if } i \in \mathcal{J} \\ 1 & \text { if } i \in \mathcal{B}\end{cases}
$$

where $\alpha^{\prime}=\liminf \operatorname{in}_{k \rightarrow \infty} \alpha^{k}$. Therefore as long as there is at least one limit point $\alpha^{\prime}$ of $\left\{\alpha^{k}\right\}$ that is not very small, the sequences $\left\{T\left(x_{i}^{k}\right)\right\}$ accumulates around three reasonably distinguished values $1-\bar{\alpha}, 1-\frac{\bar{\alpha}}{2}$, and 1 corresponding to variables with indices in $\mathcal{N}, \mathcal{J}$, and $\mathcal{B}$ respectively. This shows that the Tapia indicator sequence gives a reasonable identification criterion even if the steplength sequence does not converge. On the other hand, a better convergence property can be obtained if the definition of the Tapia indicator is slightly modified changed as follows

$$
T\left(x_{i}^{k}\right)=\left|\frac{x_{i}^{k}+\Delta x_{i}^{k}}{x_{i}^{k}}\right|,
$$


and

$$
T\left(y_{i}^{k}\right)=\left|\frac{y_{i}^{k}+\Delta y_{i}^{k}}{y_{i}^{k}}\right| .
$$

In this case we have

$$
\lim _{k \rightarrow \infty} T\left(x_{i}^{k}\right)= \begin{cases}0 & \text { if } i \in \mathcal{N} \\ 1 / 2 & \text { if } i \in \mathcal{J} \\ 1 & \text { if } i \in \mathcal{B}\end{cases}
$$

\section{Remarks:}

- The convergence rate results (3.2) and (3.3) also hold for $T_{i}\left(y^{k}\right)$ for $i \in \mathcal{N}$.

- Assume that $\sigma^{k}=O\left[\left(x^{k}\right)^{T} y^{k}\right]$ in Algorithm 2.1. From (3.2), (3.3), and Theorem 2.6 of Monteiro and Wright [10] we conclude that the convergence rate of the Tapia indicators, for $i \in \mathcal{B} \cup \mathcal{N}$, is at least $\mathrm{R}$-linear. This result is weaker than the rate obtained by El-Bakry, Tapia, and Zhang [2]. As we will see in the numerical experimentation, although the Tapia indicators demonstrate a slow local rate of convergence, they give a reasonable early identification of the status of the variables.

The following corollary is a direct result of the Lemma 3.1 and concerns with the convergence and rate of convergence of both the variables used as indicators and the primal-dual indicator.

Corollary 3.1 Let $\left\{\left(x^{k}, y^{k}\right)\right\}$ be a sequence generated by Algorithm 2.1 such that Assumptions (A1)-(A2) hold. Assume further that

1. the centering parameter is chosen such that $\sigma^{k} \rightarrow 0$.

2. $\lim \alpha^{k} \rightarrow \bar{\alpha} \in(0,1]$.

Then for all $i \in \mathcal{N}$, both the variables as indicator $V(x)$ and the primal-dual indicator PD $(x, y)$ converges to zero $Q$-linearly with $Q_{1}$-factor $1-\hat{\alpha}$, and for all $i \in \mathcal{J}, V(x)$ converges to zero Q-linearly with $Q_{1}$-factor $1-\frac{\hat{\alpha}}{2}$

Moreover, if $\alpha^{k} \rightarrow 1$ then for $i \in \mathcal{N}$, both $V(x)$ and $P D(x, y)$ converges to zero Q-superlinearly, and for all $i \in \mathcal{J}, V(x)$ converges to zero Q-linearly with $Q_{1}$-factor $\frac{1}{2}$.

Proof: The proof follows from Lemma 3.1 and the fact that the Tapia indicator $T_{i}\left(x^{k}\right)$ is the $Q_{\mathrm{I}}$-factor for both $\left\{x_{i}^{k}\right\}$ and $\left\{x_{i}^{k} / y_{i}^{k}\right\}$.

It is worth mentioning that while the variables used as indicators converge for variables with indices in $\mathcal{J}$, the convergence of the primal-dual indicator has not been yet established for these variables. The best that has been proved for those variables is that their primal-dual indicators are bounded. This conclusion follows directly from Lemma 2.4 of Monteiro and Wright [10]. This 
fact lead Monteiro and Wright [10] to propose the use of the primal-dual indicators only to predict members of $\mathcal{N}$ and $\mathcal{B}$ in their finite termination technique. An example of the behavior of the three indicators is given in the next section. The example actually suggests that the primal-dual indicators for variables in $\mathcal{J}$, if they converge, do not converge to meaningful limits that can be used early in the algorithm to detect the existence of variables $\left(x_{i}, y_{i}\right), i \in \mathcal{J}$.

Montiero and Wright [10] proved that the convergence rate of $\left\{\left(x^{k}\right)^{T} y\right\}$ is at most Q-linear if $\mathcal{J} \neq \emptyset$. They also note that the $Q_{1}$-factor in this case is less or equal $1 / 4$ if $\alpha^{k} \rightarrow 1$. A final result concerning the rate of convergence of Algorithm 2.1 follows from Lemma 3.1, and is given in the following proposition. This result states that the best $Q_{1}$-factor that can be obtained in this case is $1 / 4$.

Proposition 3.1 Let $\left\{\left(x^{k}, y^{k}\right)\right\}$ be a sequence generated by Algorithm 2.1 such that Assumptions (A1)-(A2) hold. Assume further that

1. $\mathcal{J} \neq \emptyset$.

2. the centering parameter is chosen such that $\sigma^{k} \rightarrow 0$.

3. $\lim _{k \rightarrow \infty} \alpha^{k} \rightarrow 1$.

Then the the sequence $\left\{\left(x^{k}\right)^{T} y^{k}\right\}$ converges to zero Q-linearly with $Q_{1}$-factor $1 / 4$.

Proof: From Theorem 4.1 in Monteiro and Wright [10], the $Q_{1}$-factor of $\left\{\left(x^{k}\right)^{T} y^{k}\right\}$ is given by

$$
Q_{1}=\lim _{k \rightarrow \infty} \sup \left[1-\alpha^{k}+\frac{\left(\alpha^{k}\right)^{2}}{4} \frac{\left(x_{J}^{k}\right)^{T} y_{J}^{k}}{\left(x^{k}\right)^{T} y^{k}},\right]
$$

which implies, under Condition 3, that

$$
Q_{1} \leq \frac{1}{4}
$$

From Lemma 3.1, we have

$$
\lim _{k \rightarrow \infty} \frac{x_{i}^{k+1} y_{i}^{k+1}}{x_{i}^{k} y_{i}^{k}}=\frac{1}{4} \text { for all } i \in \mathcal{J}
$$

Consider the $Q_{1}$-factor of the sequence $\left\{\theta_{\mathcal{J}}^{k}\right\}$ where $\theta_{\mathcal{J}}^{k}=\sum_{i \in \mathcal{J}} x_{i}^{k} y_{i}^{k}$.

$$
\begin{aligned}
Q_{1}\left(\left\{\theta_{\mathcal{J}}^{k}\right\}\right) & \equiv \lim \sup _{k \rightarrow \infty} \frac{\sum_{i \in \mathcal{J}} x_{i}^{k+1} y_{i}^{k+1}}{\sum_{i \in \mathcal{J}} x_{i}^{k} y_{i}^{k}} \\
& =\lim \sup _{k \rightarrow \infty}\left[\sum_{i \in \mathcal{J}}\left(\sum_{j \neq i \in \mathcal{J}} x_{j}^{k} y_{j}^{k}\right)^{-1} \frac{x_{i}^{k+1} y_{i}^{k+1}}{x_{i}^{k} y_{i}^{k}}\right] /\left[\sum_{i \in \mathcal{J}}\left(\sum_{j \neq i \in \mathcal{J}} x_{j}^{k} y_{j}^{k}\right)^{-1}\right], \\
& \geq \lim _{k \rightarrow \infty} \min _{i \in \mathcal{J}}\left(\frac{x_{i}^{k+1} y_{i}^{k+1}}{x_{i}^{k} y_{i}^{k}}\right)=\frac{1}{4},
\end{aligned}
$$


since all the sequences $\left\{\frac{x_{i}^{k+1} y_{i}^{k+1}}{x_{i}^{k} y_{i}^{k}}\right\}$, for $i \in \mathcal{J}$, converges to $1 / 4$ by (3.7). It is also clear from Lemma 3.1 that

$$
\theta_{\mathcal{B} \cup \mathcal{N}}^{k} \rightarrow 0 \text { q-superlinearly }
$$

where

$$
\theta_{\mathcal{B} \cup \mathcal{N}}^{k}=\sum_{i \in \mathcal{B} \cup \mathcal{N}} x_{i}^{k} y_{i}^{k}
$$

Now consider the $Q_{1}$-factor of $\left\{\left(x^{k}\right)^{T} y^{k}\right\}$,

$$
\begin{aligned}
Q_{1}\left(\left\{\left(x^{k}\right)^{T} y^{k}\right\}\right) & \equiv \lim \sup _{k \rightarrow \infty} \frac{\sum_{\mathcal{L}=1} n x_{i}^{k+1} y_{i}^{k+1}}{\sum_{i=1} n x_{i}^{k} k_{i}^{k}} \\
& =\lim \sup _{k \rightarrow \infty} \frac{\theta_{\mathcal{J}}^{k+1}+\theta_{\mathcal{B} \cup \mathcal{N}}^{k+1}}{\theta_{\mathcal{J}}^{k}+\theta_{\mathcal{B} \cup \mathcal{N}}^{k}} \\
& \geq \lim \sup _{k \rightarrow \infty} \frac{\theta_{\mathcal{J}}^{k+1}}{\theta_{\mathcal{J}}^{k}} /\left(1+\frac{\theta_{\mathcal{B} \cup \mathcal{N}}^{k}}{\theta_{\mathcal{J}}^{k}}\right) .
\end{aligned}
$$

Since $\left\{\boldsymbol{\theta}_{\mathcal{J}}^{k}\right\}$ converges to zero Q-linearly with $Q_{1}$-factor at least $1 / 4$, then for sufficiently large $k$ we have

$$
\frac{\theta_{\mathcal{J}}^{k+1}}{\theta_{\mathcal{J}}^{k}} \geq \tau>0
$$

which implies that

$$
\frac{\theta_{\mathcal{B} \cup \mathcal{N}}^{k}}{\theta_{\mathcal{J}}^{k}} \rightarrow 0
$$

Therefore

$$
Q_{1}\left(\left\{\left(x^{k}\right)^{T} y^{k}\right\}\right) \geq \frac{1}{4}
$$

From (3.6) and the last relation, we obtain the desired result.

This proposition asserts that, for degenerate monotone linear complementarity problems, the best $Q_{1}$-factor of the sequence $\left\{\left\|F\left(x^{k}, y^{k}\right)\right\|_{1}\right\}$, where $\left\{\left(x^{k}, y^{k}\right)\right\}$ is generated by Algorithm 2.1. is 1/4. The existence of even one pair $\left(x_{j}, y_{j}\right), j \in \mathcal{J}$ adversely slows down the algorithm regardless of how fast other variables approach zero. This fact will be numerically demonstrated in the next section.

\section{Numerical Example}

In the following experiment, a perturbed Newton interior-point algorithm is used. The algorithm is coded in $M A T L A B$. A problem is said to be solved to an accuracy of $10^{-d}$, for some positive integer $\mathrm{d}$, if the algorithm stops with $\left\|F\left(x^{k}, y^{k}\right)\right\|_{2} \leq 10^{-d}$. The problem that is considered here is solved to an accuracy of $10^{-8}$. The parameter $\sigma^{k}$ in Algorithm 2.1 is chosen such that $\sigma^{k} \rightarrow 0$. The iterates generated by the code are not necessarily feasible with respect to the linear equations 
$M x-y+q$. The experiments were performed on a Sun SPARCstation 10 Model 41 with 128 Megabytes of memory running SunOS 4.1.3.

An example of a problem with a solution that does not satisfy strict complementarity is the following

$$
\begin{array}{ll}
\text { minimize } & \left(x_{1}+3 x_{2}+x_{3}\right)^{2}+4\left(x_{1}+x_{2}\right)^{2} \\
\text { subject to } & 1-x_{1}-x_{2}-x_{3}=0 \\
& 6 x_{2}+4 x_{3}-x_{1}^{3}-x_{4}-3=0 \\
& \left(x_{1}, x_{2}, x_{3}, x_{4}\right) \geq 0
\end{array}
$$

This is problem number 32 in the Hock and Schitkwoski [7] set of test problems. Figure 2.1

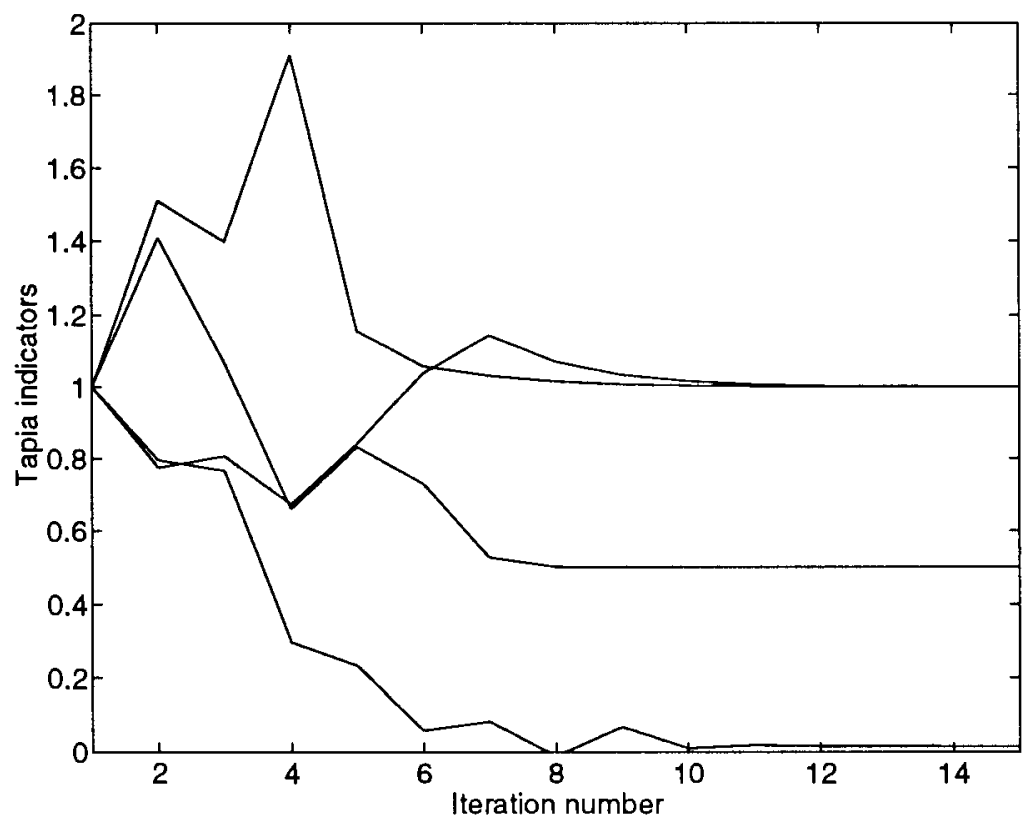

Figure 2: The Tapia indicator for all variables in problem \#32.

demonstrates the behavior of the Tapia indicators for the all variables in the problem. We note that $x_{4}$ is a slack variable added to the original inequality constraints. The algorithm gives the point $\left(6.26 \times 10^{-5}, 0,0.999,0.997\right)$ as an approximate solution to the problems. The Lagrange multipliers corresponding to the inequality constraints are $\left(5.01 \times 10^{-4}, 3.999,0,0\right)$. Hence both $x_{1}$ and the corresponding multiplier are zero at the solution. We note that for this example we have $\alpha^{k} \rightarrow 1$. The behavior of the Tapia indicator predicted by Lemma 3.1 is clearly clemonstrated where the Tapia indicator corresponding to $x_{1}$ converges to 0.5 .

A comparison between the three indicators is given in Table 1 for this problem. The first column gives the iteration number. The second column gives the Tapia indicator for the variable 


\begin{tabular}{|c|c|c|c|c|c|}
\hline Iteration number & $T_{1}\left(x^{k}\right)$ & $T_{1}\left(y^{k}\right)$ & $x_{1}^{k}$ & $y_{1}^{k}$ & $x_{1}^{k} / y_{1}^{k}$ \\
\hline \hline 1 & $1.4096 \mathrm{e}+00$ & $1.8187 \mathrm{e}+00$ & $1.0000 \mathrm{e}-01$ & $1.9791 \mathrm{e}+01$ & $5.0528 \mathrm{e}-03$ \\
2 & $1.0678 \mathrm{e}+00$ & $6.3976 \mathrm{e}-02$ & $1.4096 \mathrm{e}-01$ & $3.5994 \mathrm{e}+01$ & $3.9161 \mathrm{e}-03$ \\
3 & $6.5954 \mathrm{e}-01$ & $5.1126 \mathrm{e}-01$ & $1.4882 \mathrm{e}-01$ & $8.2617 \mathrm{e}+00$ & $1.8013 \mathrm{e}-02$ \\
4 & $8.3130 \mathrm{e}-01$ & $3.1037 \mathrm{e}-01$ & $9.8153 \mathrm{e}-02$ & $4.2239 \mathrm{e}+00$ & $2.3238 \mathrm{e}-02$ \\
5 & $7.3057 \mathrm{e}-01$ & $3.7558 \mathrm{e}-01$ & $8.1708 \mathrm{e}-02$ & $1.3310 \mathrm{e}+00$ & $6.1391 \mathrm{e}-02$ \\
6 & $5.2704 \mathrm{e}-01$ & $5.0405 \mathrm{e}-01$ & $5.9693 \mathrm{e}-02$ & $4.9987 \mathrm{e}-01$ & $1.1942 \mathrm{e}-01$ \\
7 & $5.0107 \mathrm{e}-01$ & $5.0053 \mathrm{e}-01$ & $3.1461 \mathrm{e}-02$ & $2.5196 \mathrm{e}-01$ & $1.2486 \mathrm{e}-01$ \\
8 & $5.0006 \mathrm{e}-01$ & $5.0004 \mathrm{e}-01$ & $1.6002 \mathrm{e}-02$ & $1.2803 \mathrm{e}-01$ & $1.2499 \mathrm{e}-01$ \\
9 & $5.0001 \mathrm{e}-01$ & $5.0000 \mathrm{e}-01$ & $8.0022 \mathrm{e}-03$ & $6.4018 \mathrm{e}-02$ & $1.2500 \mathrm{e}-01$ \\
10 & $5.0000 \mathrm{e}-01$ & $5.0000 \mathrm{e}-01$ & $4.0084 \mathrm{e}-03$ & $3.2067 \mathrm{e}-02$ & $1.2500 \mathrm{e}-01$ \\
11 & $5.0000 \mathrm{e}-01$ & $5.0000 \mathrm{e}-01$ & $2.0042 \mathrm{e}-03$ & $1.6034 \mathrm{e}-02$ & $1.2500 \mathrm{e}-01$ \\
12 & $5.0000 \mathrm{e}-01$ & $5.0000 \mathrm{e}-01$ & $1.0021 \mathrm{e}-03$ & $8.0168 \mathrm{e}-03$ & $1.2500 \mathrm{e}-01$ \\
13 & $5.0000 \mathrm{e}-01$ & $5.0000 \mathrm{e}-01$ & $5.0105 \mathrm{e}-04$ & $4.0084 \mathrm{e}-03$ & $1.2500 \mathrm{e}-01$ \\
14 & $5.0000 \mathrm{e}-01$ & $5.0000 \mathrm{e}-01$ & $2.5053 \mathrm{e}-04$ & $2.0042 \mathrm{e}-03$ & $1.2500 \mathrm{e}-01$ \\
15 & $5.0000 \mathrm{e}-01$ & $5.0000 \mathrm{e}-01$ & $1.2526 \mathrm{e}-04$ & $1.0021 \mathrm{e}-03$ & $1.2500 \mathrm{e}-01$ \\
\hline
\end{tabular}

Table 1: Several indicators in the absence of strict complementarity

$x_{1}$ while the third gives the Tapia indicator for the corresponding multiplier $y_{1}$. The fourth and fifth columns give the value of the variables $x_{1}$ and $y_{1}$ respectively, while the sixth column gives the value of the primal-dual indicator $x_{1} / y_{1}$. From the table we can see that although the variables $x_{1}$ and $y_{1}$ both converges to zero, none of them is "sufficiently" small until iteration 10 . In fact both $x_{1}$ and $y_{1}$ are not small enough even when the algorithm terminates. On the other hand, although the primal-dual indicator $x / y$ seems to converge to 0.125 , its terminal value is meaningless. On the contrary, both Tapia indicators converge, as the theory predicts, to 0.5 . Observe also that they converge relatively early (starting from iteration 6 ). We note here that at iteration 6 , the residual $\|M x-y+q\|$ is $10^{-4}$, i.e. the iterates are close to the feasibility region.

Observe that, in Figure 2, the Tapia indicator corresponding to the zero variable $x_{2}$ is small but not close to zero. This is due to the fact that the Tapia indicator corresponding to this variable converges to zero slowly, as predicted by the theory. Observe, however, that it gives an early identification of the states of $x_{2}$ at the solution. In Table 2 the Tapia indicator corresponding to $x_{2}$ is given. The first column gives the iteration number (the code terminated successfully at iteration $15)$ for the last 6 iterations. The second column gives the Tapia indicator for the zero variable when $\sigma=O\left(\left\|F\left(x^{k}, y^{k}\right)\right\|_{2}^{2}\right)$. It is clear that the convergence of the Tapia indicator to zero is slow. The third column gives the values of the same indicator when $\sigma=O\left(\left\|F\left(x^{k}, y^{k}\right)\right\|_{2}^{4}\right)$. The convergence to zero is obviously faster. In fact it seems that the Tapia indicator $T_{2}\left(x^{k}\right)$ convergence to zero linearly 
with a $Q_{1}$-factor close to 0.50 . This means that zero components, either in $x$ or $y$, converges faster to zero. It is interesting, however, to observe that, the algorithm itself was still slow (it took the same number of iteration in both cases). Another interesting point to observe is that in both cases the $Q_{1}$-factor of the residual sequence is approximately $1 / 4$, see columns 4 and 7 . Other runs with tighter stopping criterion also support our observation. In all cases the best $Q_{1}$-factor we obtained was $1 / 4$. In all cases $\alpha^{k} \rightarrow 1$. This performance is predicted by Proposition 3.1.

\begin{tabular}{|c|c|c|c|c|c|c|}
\hline \multirow{2}{*}{$\begin{array}{c}\text { Iteration } \\
\text { number }\end{array}$} & \multicolumn{3}{|c|}{$\sigma^{k}=\left\|F\left(x^{k}, y^{k}\right)\right\|_{2}^{2}$} & \multicolumn{3}{c|}{$\sigma^{k}=\left\|F\left(x^{k}, y^{k}\right)\right\|_{2}^{4}$} \\
\cline { 2 - 7 } & $\begin{array}{c}\text { relative } \\
\text { residual }\end{array}$ & $\begin{array}{c}\text { Tapia } \\
\text { indicator }\end{array}$ & $\begin{array}{c}\text { Q1-factor } \\
\text { of residual }\end{array}$ & $\begin{array}{c}\text { relative } \\
\text { residual }\end{array}$ & $\begin{array}{c}\text { Tapia } \\
\text { Indicator }\end{array}$ & $\begin{array}{c}\text { Q1-factor } \\
\text { of residual }\end{array}$ \\
\hline \hline 10 & $3.21 \mathrm{e}-05$ & $1.9410 \mathrm{e}-02$ & $2.4884 \mathrm{e}-01$ & $4.90 \mathrm{e}-05$ & $4.9492 \mathrm{e}-03$ & $2.5521 \mathrm{e}-01$ \\
11 & $8.03 \mathrm{e}-06$ & $1.6790 \mathrm{e}-02$ & $2.5016 \mathrm{e}-01$ & $1.24 \mathrm{e}-05$ & $2.4869 \mathrm{e}-03$ & $2.5306 \mathrm{e}-01$ \\
12 & $2.01 \mathrm{e}-06$ & $1.6458 \mathrm{e}-02$ & $2.5031 \mathrm{e}-01$ & $3.11 \mathrm{e}-06$ & $1.2465 \mathrm{e}-03$ & $2.5081 \mathrm{e}-01$ \\
13 & $5.02 \mathrm{e}-07$ & $1.6060 \mathrm{e}-02$ & $2.4975 \mathrm{e}-01$ & $7.78 \mathrm{e}-07$ & $6.2397 \mathrm{e}-04$ & $2.5016 \mathrm{e}-01$ \\
14 & $1.26 \mathrm{e}-07$ & $1.5854 \mathrm{e}-02$ & $2.5100 \mathrm{e}-01$ & $1.95 \mathrm{e}-07$ & $3.1208 \mathrm{e}-04$ & $2.5064 \mathrm{e}-01$ \\
15 & $3.14 \mathrm{e}-08$ & $1.5742 \mathrm{e}-02$ & $2.4921 \mathrm{e}-01$ & $4.87 \mathrm{e}-08$ & $1.5605 \mathrm{e}-04$ & $2.4974 \mathrm{e}-01$ \\
\hline
\end{tabular}

Table 2: Tapia indicator for two choices of $\sigma^{k}$

\section{Conclusion}

The convergence and the convergence rate of the Tapia indicators for feasible-iterate interior-point methods for monotone linear complementarity problems is established when strict complementarity does not hold. It is shown that the Tapia indicators converge at least R-linearly to their terminal value. The convergence rate for the variables used as indicators and the primal-dual indicator corresponding to zero variables is also established. Another result concerning the behavior of interior-point methods in the presence of solution that do not satisfy strict complementarity follows form Proposition 3.1. This proposition asserts that the best $Q_{1}$-factor of the sequence $\left\{\left(x^{k}\right)^{T} y^{k}\right\}$ is $1 / 4$. The convergence of the Tapia indicators for infeasible-iterate methods is still under investigation.

\section{References}

[1] A. S. El-Bakry. On the role of indicators in identifying zero variables in linear programming. PhD thesis, Department of Mathematical Sciences, Rice University, Houston, Texas 77251, 1991. 
[2] A. S. El-Bakry, R. A. Tapia, and Y. Zhang. A study of indicators for identifying zero variables in interior-point methods. To appear in SIAM Review, 1994.

[3] D. M. Gay. Electronic mail distribution of linear programming test problems. COAL Newsletter, 13:10-12, 1985.

[4] D. M. Gay. Stopping tests that compute optimal solutions for interior-point linear programming algorithms. In S. Gomez, J. P. Hennart, and R. A. Tapia, editors, Advances in Numerical Partial Differential Equations and Optimization : Proceedings of the Fifth Mexico-United Staets Workshop, volume 47 of Proceedings in Applied Mathematics, pages 17-42. SIAM Publications, Philadelphia, PA, USA, 1991.

[5] R. Glowinski and A. J. Kearsley. On the simulation and control of some friction constrained motions. Technical Report TR93-21, Dept. of Computational and Applied Mathematics, Rice University, Houston, Texas 77251, 1993.

[6] A. J. Goldman and A. W. Tucker. Theory of linear programming. In H. W. Kuhn and A. W. Tucker, editors, Linear inequalities and related systems, pages 53-97. Princeton University Press, 1956.

[7] W. Hock and K. Schittkowski. Test Examples for Nonlinear Programming Codes. SpringerVerlag, New York, 1981.

[8] I. J. Lustig. An implementation of a strongly polynomial time algorithm for basis recovery. Dept. Civil Eng. and O.R., Princeton University, in preparation.

[9] S. Mehrotra. On finding a vertex solution using interior point methods. Linear Algebra and Its Applications, 152:233-253, 1991.

[10] R.C. Monteiro and S. Wright. Local convergence of interior-point algorithms for degenerate monotone lcp problems. Technical report, Mathematics and Computer Science Division, Argonne National Laboratory, 1993. MSC-P357-0493.

[11] R. A. Tapia. On the role of slack variables in quasi-Newton methods for constrained optimization. In L. C. W. Dixon and G. P. Szegö, editors, Numerical Optimization of dynamic systems, pages 235-246. North-Holland, 1980.

[12] Y. Ye. On the finite convergence of interior-point algorithms for linear programming. TechnicaI Report 91-05, Dept. of Management Sciences, University of Iowa, Iowa City, IA 52242, USA, 1991. To appear in Mathematical Programming Ser. B. 